\title{
Association between Polymorphisms of X-Ray Repair Cross Complementing Group 1 Gene and Pancreatic Cancer Risk: a Systematic Review with Meta-Analysis
}

\author{
Jun Chen ${ }^{1} \cdot$ Hong Wang ${ }^{1} \cdot$ Zhiming $\mathrm{Li}^{2}$
}

Received: 17 August 2017 / Accepted: 8 November 2017 /Published online: 28 December 2017

(C) The Author(s) 2017. This article is an open access publication

\begin{abstract}
Emerging evidences have shown that common genetic polymorphisms in X-ray repair cross complementing group 1 (XRCC1) gene may be associated with the development of pancreatic cancer, but individually published studies and previous meta-analyses revealed inconclusive results. The aim of our study was to investigate the association between polymorphisms in XRCC1 gene and pancreatic cancer risk. We conducted a search of PubMed, Embase, the Cochrane Library and Web of Science databases. Odds ratios (ORs) with 95\% confidence intervals (CIs) were determined as measures of the strength of association between polymorphisms of $\mathrm{XRCC} 1$ and pancreatic cancer risk. Sensitivity analysis and publication bias were evaluated. All analyses were undertaken using the STATA 13.0. A total of 10 studies were included in this systematic review. Five common functional singlenucleotide polymorphisms (SNPs) in XRCC1 gene were found, including Arg399Gln G > A (rs25487), Arg194Trp C $>$ T (rs1799782), Arg280His G > A (rs25489), c.1517G > C, c.1471G $>$ A. Results from our stratified analysis based on Hardy-Weinberg equilibrium (HWE) showed that there was a robust significant association between Arg280His polymorphism and pancreatic cancer risk (allelic model, OR 0.743,
\end{abstract}

Electronic supplementary material The online version of this article (https://doi.org/10.1007/s12253-017-0364-6) contains supplementary material, which is available to authorized users.

Jun Chen

chen_jun@hainan.net

1 Department of General Surgery, Affiliated Hospital of Traditional Chinese Medicine, Xinjiang Medical University, No. 116 the Yellow River Road, Urumqi 830000, China

2 Department of Surgery, Shihezi Hospital of traditional Chinese Medicine, Shihezi, China
95\% CI $0.576-0.958, P=0.022$; heterozygous model, OR $0.701,95 \%$ CI $0.525-0.936, P=0.016$; dominant model, OR $0.710,95 \%$ CI $0.537-0.939, P=0.016$ ). We also found a statistically significant association between c. $1517 \mathrm{G}>\mathrm{C}$ polymorphism and pancreatic cancer risk (Allelic model, OR $1.252,95 \%$ CI 1.064-1.473, $P=0.007$ ). No significant results were obtained for Arg399Gln, Arg194Trp and c.1471G > A polymorphisms. The present meta-analysis suggested that Arg280His and c. $1517 \mathrm{G}>\mathrm{C}$ polymorphisms in XRCC1 gene were associated with pancreatic cancer risk.

Keywords XRCC1 $\cdot$ Polymorphisms · Pancreatic cancer · Meta-analysis

\section{Introduction}

Pancreatic cancer, although infrequent, has an exceptionally high mortality rate. The development of pancreatic cancer is a complex and multifactorial process [1], and has many associated risk factors, such as include high-fat diet, smoking, chronic pancreatitis, primary sclerosing cholangitis, hereditary pancreatitis, family history of pancreatic cancer and diabetes mellitus [2]. It is well known that the genetic factors play key roles in the development of pancreatic cancer [1].

DNA repair systems are essential for maintaining the integrity of the genome and play key roles in protecting against mutations. The single nucleotide polymorphisms (SNPs) in DNA repair genes can cause the differences in the DNA repair capacity, which may contribute to the development of cancer because reduced DNA repair capacity may lead to genetic instability and carcinogenesis [3, 4]. It has been well accepted that the SNPs in the base excision repair (BER) gene can change the individual repair capacity in response to DNA damage [5]. X-ray repair cross-complementation group 1 
(XRCC1) gene that is located on chromosome no. 19q13.213.3 plays a critical role in the BER of DNA damage [6]. There are more than 300 validated SNPs in the XRCC1 gene reported in the dbSNP database (http://www.ncbi.nlm.nih. gov/SNP/). Several SNPs in XRCC1 are associated with several types of cancer cancers risk, such as breast cancer [7], hepatocellular carcinoma [8] and lung cancer [9]. Recently, several studies have investigated the association between SNPs in XRCC1 gene and pancreatic cancer risk. However, results remain controversial in part because of the differences from the sample sizes. In this study, five common functional single-nucleotide polymorphisms (SNPs) in XRCC1 gene were found, including Arg399Gln G > A (rs25487), Arg194Trp C > T (rs1799782), Arg280His G > A (rs25489), c.1517G > C, c.1471G > A. For better understanding of the effects of the five SNPs in XRCC1 gene on pancreatic cancer risk, we conducted a systematic review with metaanalysis of previous published studies.

\section{Materials and Methods}

\section{Identification of Eligible Studies}

This meta-analysis was conducted according to PRISMA statement [10] (S1 PRISMA Checklist) and Cochrane Collaboration guidelines (http://handbook.cochrane.org/). We performed a systematic search using PubMed, Embase, the Cochrane Library and Web of Science with the last search updated on February 12, 2017. The search strategies were based on combinations of the following terms: "XRCC1 or rs25487 or Arg399Gln or rs1799782 or Arg194Trp or rs25489 or Arg280His or c.1517G $>\mathrm{C}$ or c.1471G $>$ A" AND "pancreatic cancer or pancreatic carcinoma". In addition, all references of included studies and previously published reviews were manually screened and reviewed for relevant studies.

\section{Eligibility Criteria}

Studies were included in this meta-analysis if they satisfied the following criteria: (1) It was a case-control study; (2) The study assessed the association between XRCC1 gene (Arg399Gln, Arg194Trp, Arg280His, c.1517G > C, or c. $1471 \mathrm{G}>\mathrm{A}$ ) polymorphisms and pancreatic cancer risk; (3) The study provided complete genotypes distribution data for estimating the odds ratios (ORs) and $95 \%$ confidence intervals (95\% CIs); (4) Genotyping method in each study was universally acknowledged. The exclusion criteria of studies were as follows: (1) duplication of the previous publications, (2) abstract, comment, case reports, letters, and review, and (3) no sufficient data were provided. Two reviewers independently identified eligible studies according to the selection criteria. Disagreements were resolved by reaching a consensus among all authors.

\section{Data Extraction}

Data were extracted by two reviewers independently, and then another reviewer verified them and any discrepancies were resolved by consensus. The following data were extracted from each study: the first author's name, publication year, country of origin, ethnicity, genotyping methods, numbers of cases and controls, genotype frequency in cases and controls, respectively.

\section{Statistical Analysis}

Hardy-Weinberg equilibrium (HWE) was evaluated by Pearson's goodness-of-fit $\chi^{2}$ test for each study in the controls, and a $P$ value of less than 0.05 was considered as deviation from HWE [11]. The strength of the association between the $\mathrm{XRCC} 1$ gene polymorphisms and pancreatic cancer risk was assessed by calculating crude odds ratios (ORs) along with their 95\% confidence intervals (CIs). The pooled ORs (Arg399Gln, Arg280His and c.1471G > A polymorphisms) were calculated for the allelic model (A versus $G$ ), heterozygous model (GA versus GG), homozygous model (AA versus GG), dominant model (AA + GA versus GG) and recessive model (AA versus GA + GG). The pooled ORs (Arg194Trp polymorphism) were calculated for the allelic model ( $T$ versus $\mathrm{C})$, heterozygous model (CT versus CC), homozygous model (TT versus $\mathrm{CC}$ ), dominant model $(\mathrm{TT}+\mathrm{CT}$ versus $\mathrm{CC}$ ) and recessive model (TT versus $\mathrm{CT}+\mathrm{CC}$ ). Similarly, the pooled ORs (c. $1517 \mathrm{G}>\mathrm{C}$ polymorphism) were calculated for the allelic model ( $\mathrm{C}$ versus $\mathrm{G}$ ), heterozygous model ( $\mathrm{GC}$ versus $\mathrm{GG})$, homozygous model (CC versus GG), dominant model $(\mathrm{CC}+\mathrm{GC}$ versus $\mathrm{GG})$ and recessive model $(\mathrm{CC}$ versus $\mathrm{GC}+$ $\mathrm{GG})$. Heterogeneity was determined using the Cochrane's $\mathrm{Q}$ test and $\mathrm{I}^{2}$ statistics [12]. When the Q-test showed a $P<0.05$ or $\mathrm{I}^{2}$ test exhibited $>50 \%$, the random-effect model was used for the meta-analysis [13]; otherwise, the fixed-effect model was chosen [14]. Sensitivity analyses were also carried out by sequential omission of each study one at a time to ensure the stability of our results. Publication bias was assessed using Begg's funnel plot [15] and Egger's linear regression method [16]. A $P$ value of less than 0.05 was considered representative of statistically significant publication bias. All statistical analyses were performed by using STATA version 13.0.

\section{Results}

\section{Study Characteristics}

We give the detailed search strategies and results in the supplemental material (see S1 Table in the supplemental 
material). A total of 58 records were initially identified from PubMed, Embase, the Cochrane Library and Web of Science. Based on the eligibility criteria, a total of 10 studies ( 8 studies in English and 2 in Chinese) were included in this systematic review. In the end, 9 case-control studies for Arg399Gln polymorphism, 7 case-control studies for Arg194Trp, 3 casecontrol studies for Arg280His, 2 case-control studies for c. $1517 \mathrm{G}>\mathrm{C}$, and 2 case-control studies for c. $1471 \mathrm{G}>\mathrm{A}$ were selected for this meta-analysis. The flow diagram summarizing the literature review process and final participation is shown in Fig. 1. The main characteristics of the selected studies are presented in Table 1.

\section{Meta-Analysis of Data on the Arg399GIn Polymorphism}

The meta-analysis of a possible association between the XRCC1 gene polymorphisms and pancreatic cancer risk was summarized in Table 2. For XRCC1 gene Arg399Gln polymorphism, 9 case-control studies including 2067 cases and 4426 controls were included in the meta-analysis. Based on the total populations, none of the five genetic models indicated a significant association (allelic model, OR 1.111, 95\% CI 0.954-1.293, $P=0.177$; heterozygous model, OR 1.083,
95\% CI 0.961-1.220, $P=0.190$; homozygous model, OR 1.212 , 95\% CI 0.906-1.620, $P=0.195$; dominant model, OR $1.123,95 \%$ CI $0.939-1.342, P=0.204$; recessive model, OR $1.135,95 \%$ CI $0.950-1.356, P=0.162$ ). We performed a stratified analysis based on ethnicity, and no significant association was observed between the Arg399Gln polymorphism and pancreatic cancer risk in Asians or Caucasians.

\section{Meta-Analysis of Data on the Arg194Trp Polymorphism}

For XRCC1 gene Arg194Trp polymorphism, 7 case-control studies including 1594 cases and 3517 controls were included in the meta-analysis. There was no significant association between XRCC1 gene Arg194Trp polymorphism and pancreatic cancer risk (allelic model, OR 1.223, 95\% CI 0.939-1.592, $P=0.136$; heterozygous model, OR 1.229 , 95\% CI $0.881-$ $1.713, P=0.225$; homozygous model, OR $1.140,95 \% \mathrm{CI}$ 0.832-1.563, $P=0.414$; dominant model, OR $1.247,95 \%$ CI $0.900-1.726, P=0.184$; recessive model, OR $1.130,95 \%$ CI $0.831-1.534, P=0.436)$. We performed a stratified analysis based on ethnicity, and no significant association was observed between the Arg194Trp polymorphism and pancreatic cancer risk in Asians or Caucasians.
Fig. 1 Flowchart of study selection

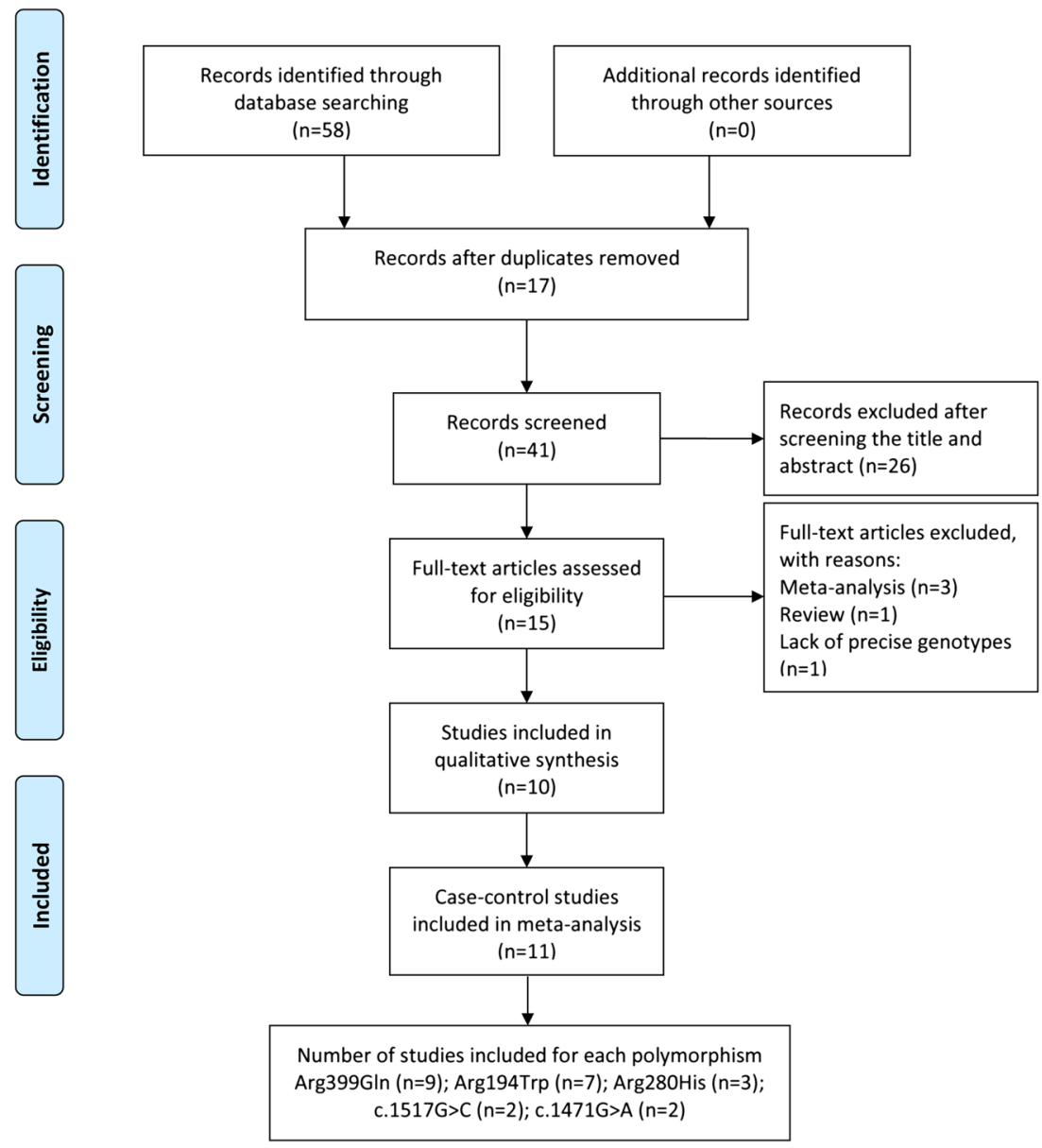


Table 1 Characteristics of studies included in the meta-analysis

\begin{tabular}{|c|c|c|c|c|c|c|c|}
\hline \multirow[t]{2}{*}{ Study } & \multirow[t]{2}{*}{ Year } & \multirow[t]{2}{*}{ Country } & \multirow[t]{2}{*}{ Ethnicity } & \multirow{2}{*}{$\begin{array}{l}\text { Genotyping } \\
\text { method }\end{array}$} & \multicolumn{2}{|c|}{ Sample size } & \multirow[t]{2}{*}{ SNP } \\
\hline & & & & & Cases & Controls & \\
\hline Wang LJ & 2016 & China & Asians & PCR-RFLP & 152 & 264 & Arg399Gln; Arg280His; Arg194Trp \\
\hline Hou BH & 2016 & China & Asians & PCR-RFLP & 298 & 298 & Arg399Gln; Arg280His; Arg194Trp; c. $1517 \mathrm{G}$ > C; c.1471G > A \\
\hline Zhao ZM & 2014 & China & Asians & PCR-RFLP & 390 & 392 & c. $1517 \mathrm{G}>\mathrm{C}$ \\
\hline Yan D & 2013 & China & Asians & SNaPshot & 210 & 213 & Arg399Gln; Arg194Trp \\
\hline Chen $\mathrm{H}$ & 2013 & China & Asians & CRS-PCR & 328 & 350 & c. $1471 \mathrm{G}>\mathrm{A}$ \\
\hline Nakao M & 2013 & Japan & Asians & TaqMan & 185 & 1465 & Arg399Gln; Arg194Trp \\
\hline Mcwilliams RR & 2008 & USA & Caucasians & PCR & 481 & 625 & Arg399Gln; Arg280His; Arg194Trp \\
\hline Wang L & 2006 & China & Asians & PCR & 101 & 337 & Arg399Gln; Arg194Trp \\
\hline Jiao L & 2006 & USA & Caucasians & PCR & 384 & 357 & Arg399Gln; Arg194Trp \\
\hline Duell EJ & 2002 & USA & Caucasians & PCR-RFLP & 261 & 860 & Arg399Gln \\
\hline Duell EJ & 2002 & USA & Asians & PCR-RFLP & 17 & 53 & Arg399Gln \\
\hline
\end{tabular}

$P C R$, polymerase chain reaction; $R F L P$, restriction fragment length polymorphism; $C R S-P C R$, created restriction site-PCR; $S N P$, single nucleotide polymorphism

\section{Meta-Analysis of Data on the Arg280His Polymorphism}

For XRCC1 gene Arg280His polymorphism, 3 casecontrol studies including 918 cases and 1147 controls were included in the meta-analysis. Based on the total populations, there was no significant association between XRCC1 gene $\operatorname{Arg} 280$ His polymorphism and pancreatic cancer risk (allelic model, OR 0.956 , 95\% CI 0.589 $1.550, P=0.854$; heterozygous model, OR $0.772,95 \%$ CI $0.592-1.008, P=0.057$; homozygous model, OR $1.216,95 \%$ CI $0.630-2.349, P=0.560$; dominant model, OR $0.885,95 \%$ CI $0.565-1.385, P=0.592$; recessive model, OR $1.261,95 \%$ CI $0.653-2.432, P=0.490)$. However, based on the HWE, there was significant association between XRCC1 gene Arg280His polymorphism and pancreatic cancer risk (allelic model, OR $0.743,95 \%$ CI $0.576-0.958, P=0.022$; heterozygous model, OR 0.701, 95\% CI 0.525-0.936, $P=0.016$; dominant model, OR $0.710,95 \%$ CI $0.537-0.939, P=$ 0.016). Stratified analysis based on ethnicity was not performed due to limited studies.

\section{Meta-Analysis of Data on the C.1517G $>$ C Polymorphism}

For XRCC1 gene c.1517G $>$ C polymorphism, 2 casecontrol studies including 688 cases and 690 controls were included in the meta-analysis. There was significant association between XRCC1 gene c. $1517 \mathrm{G}>\mathrm{C}$ polymorphism and pancreatic cancer risk (allelic model, OR $1.252,95 \%$ CI $1.064-1.473, P=0.007)$. But no significant association was observed between the c. $1517 \mathrm{G}>\mathrm{C}$ polymorphism and pancreatic cancer risk under other four genetic models.

\section{Meta-Analysis of Data on the c.1471G > A Polymorphism}

For XRCC1 gene c. $1471 \mathrm{G}>\mathrm{A}$ polymorphism, 2 casecontrol studies including 626 cases and 648 controls were included in the meta-analysis. There was no significant association between XRCC1 gene c.1471G > A polymorphism and pancreatic cancer risk (allelic model, OR $0.914,95 \%$ CI $0.467-1.789, P=0.794$; heterozygous model, OR $0.948,95 \%$ CI $0.547-1.641, P=0.848$; homozygous model, OR $0.860,95 \%$ CI $0.210-3.513, P=$ 0.833; dominant model, OR $0.915,95 \%$ CI $0.444-1.886$, $P=0.810$; recessive model, OR $0.879,95 \%$ CI 0.277 2.789, $P=0.827)$.

\section{Sensitivity Analysis}

In order to assess the robustness of the meta-analysis results, we carried out a sensitivity analysis. Our results showed that pooled ORs were not materially altered, which suggested that no individual study significantly affected the pooled results.

\section{Publication Bias}

Begg's funnel plots and Egger's regression method were used to assess publication bias statistically. For the Arg399Gln polymorphism, the results of Egger's linear regression test did not provide statistical evidence of publication bias $(P>0.05)$ (Table 3). Funnel plot analyses were also not generally indicative of any strong publication bias because visual inspection of funnel plots did not show asymmetry for all comparison models, indicating the strength of the results. 
Table 2 Main results on association between XRCC1 gene polymorphisms and pancreatic cancer risk

\begin{tabular}{|c|c|c|c|c|c|c|c|c|}
\hline \multirow[t]{2}{*}{ Comparisons } & \multirow[t]{2}{*}{ No. of Studies } & \multicolumn{3}{|c|}{ Test of association } & \multirow[t]{2}{*}{ Analysis model } & \multicolumn{3}{|c|}{ Test of heterogeneity } \\
\hline & & OR & $95 \% \mathrm{CI}$ & P-value & & $x^{2}$ & P-value & $\mathrm{I}^{2}(\%)$ \\
\hline \multicolumn{9}{|c|}{ XRCC1 gene Arg399Gln polymorphism in total populations } \\
\hline Allelic (A versus G) & 9 & 1.111 & $0.954-1.293$ & 0.177 & Random & 22.70 & 0.004 & $64.8 \%$ \\
\hline Heterozygous (GA versus GG) & 9 & 1.083 & $0.961-1.220$ & 0.190 & Fixed & 12.33 & 0.137 & $35.1 \%$ \\
\hline Homozygous (AA versus GG) & 9 & 1.212 & $0.906-1.620$ & 0.195 & Random & 16.18 & 0.040 & $50.5 \%$ \\
\hline Dominant (AA + GA versus GG) & 9 & 1.123 & $0.939-1.342$ & 0.204 & Random & 18.16 & 0.020 & $55.9 \%$ \\
\hline Recessive (AA versus GA + GG) & 9 & 1.135 & $0.950-1.356$ & 0.162 & Fixed & 11.44 & 0.178 & $30.1 \%$ \\
\hline \multicolumn{9}{|c|}{ XRCC1 gene Arg399Gln polymorphism in HWE } \\
\hline Allelic (A versus G) & 8 & 1.065 & $0.921-1.230$ & 0.396 & Random & 16.38 & 0.022 & $57.3 \%$ \\
\hline Heterozygous (GA versus GG) & 8 & 1.063 & $0.939-1.203$ & 0.335 & Fixed & 11.13 & 0.133 & $37.1 \%$ \\
\hline Homozygous (AA versus GG) & 8 & 1.089 & $0.893-1.327$ & 0.400 & Fixed & 10.96 & 0.140 & $36.1 \%$ \\
\hline Dominant (AA + GA versus GG) & 8 & 1.080 & $0.902-1.292$ & 0.405 & Random & 14.79 & 0.039 & $52.7 \%$ \\
\hline Recessive (AA versus GA + GG) & 8 & 1.070 & $0.887-1.290$ & 0.481 & Fixed & 7.23 & 0.405 & $3.2 \%$ \\
\hline \multicolumn{9}{|c|}{ XRCC1 gene Arg399Gln polymorphism in Asian populations } \\
\hline Allelic (A versus G) & 6 & 1.155 & $0.900-1.483$ & 0.256 & Random & 16.81 & 0.005 & $70.2 \%$ \\
\hline Heterozygous (GA versus GG) & 6 & 1.166 & $0.985-1.382$ & 0.075 & Fixed & 7.11 & 0.213 & $29.7 \%$ \\
\hline Homozygous (AA versus GG) & 6 & 1.303 & $0.784-2.165$ & 0.307 & Random & 12.42 & 0.029 & $59.7 \%$ \\
\hline Dominant (AA + GA versus GG) & 6 & 1.180 & $0.906-1.537$ & 0.219 & Random & 12.01 & 0.035 & $58.4 \%$ \\
\hline Recessive (AA versus GA + GG) & 6 & 1.269 & $0.950-1.695$ & 0.106 & Fixed & 9.61 & 0.087 & $48.0 \%$ \\
\hline \multicolumn{9}{|c|}{ XRCC1 gene Arg399Gln polymorphism in Caucasians populations } \\
\hline Allelic (A versus G) & 3 & 1.027 & $0.917-1.151$ & 0.643 & Fixed & 3.47 & 0.177 & $42.3 \%$ \\
\hline Heterozygous (GA versus GG) & 3 & 1.007 & $0.852-1.191$ & 0.932 & Fixed & 3.74 & 0.154 & $46.5 \%$ \\
\hline Homozygous (AA versus GG) & 3 & 1.066 & $0.836-1.358$ & 0.607 & Fixed & 2.09 & 0.352 & $4.3 \%$ \\
\hline Dominant (AA + GA versus GG) & 3 & 1.038 & $0.823-1.309$ & 0.753 & Random & 4.24 & 0.120 & $52.9 \%$ \\
\hline Recessive (AA versus GA + GG) & 3 & 1.063 & $0.849-1.331$ & 0.596 & Fixed & 0.79 & 0.673 & $0.0 \%$ \\
\hline \multicolumn{9}{|c|}{ XRCC1 gene Arg194Trp polymorphism in total populations } \\
\hline Allelic (T versus $\mathrm{C}$ ) & 7 & 1.223 & $0.939-1.592$ & 0.136 & Random & 28.88 & 0.000 & $79.2 \%$ \\
\hline Heterozygous (CT versus CC) & 7 & 1.229 & $0.881-1.713$ & 0.225 & Random & 29.30 & 0.000 & $79.5 \%$ \\
\hline Homozygous (TT versus CC) & 7 & 1.140 & $0.832-1.563$ & 0.414 & Fixed & 4.83 & 0.565 & $0.0 \%$ \\
\hline Dominant (TT + CT versus CC) & 7 & 1.247 & $0.900-1.726$ & 0.184 & Random & 30.46 & 0.000 & $80.3 \%$ \\
\hline Recessive (TT versus CT + CC) & 7 & 1.130 & $0.831-1.534$ & 0.436 & Fixed & 3.33 & 0.767 & $0.0 \%$ \\
\hline \multicolumn{9}{|c|}{ XRCC1 gene Arg194Trp polymorphism in HWE } \\
\hline Allelic ( $\mathrm{T}$ versus $\mathrm{C}$ ) & 5 & 1.315 & $0.927-1.865$ & 0.125 & Random & 23.28 & 0.000 & $82.8 \%$ \\
\hline Heterozygous (CT versus CC) & 5 & 1.390 & $0.919-2.103$ & 0.118 & Random & 21.52 & 0.000 & $81.4 \%$ \\
\hline Homozygous (TT versus CC) & 5 & 1.113 & $0.758-1.635$ & 0.583 & Fixed & 3.46 & 0.483 & $0.0 \%$ \\
\hline Dominant (TT + CT versus CC) & 5 & 1.387 & $0.918-2.098$ & 0.121 & Random & 22.99 & 0.000 & $82.6 \%$ \\
\hline Recessive (TT versus CT + CC) & 5 & 1.065 & $0.733-1.546$ & 0.743 & Fixed & 2.21 & 0.698 & $0.0 \%$ \\
\hline \multicolumn{9}{|c|}{ XRCC1 gene Arg194Trp polymorphism in Asian populations } \\
\hline Allelic (T versus $\mathrm{C}$ ) & 5 & 1.080 & $0.875-1.332$ & 0.475 & Random & 9.72 & 0.045 & $58.8 \%$ \\
\hline Heterozygous (CT versus CC) & 5 & 1.048 & $0.809-1.357$ & 0.723 & Random & 8.55 & 0.073 & $53.2 \%$ \\
\hline Homozygous (TT versus CC) & 5 & 1.105 & $0.798-1.532$ & 0.547 & Fixed & 4.05 & 0.400 & $1.2 \%$ \\
\hline Dominant (TT + CT versus CC) & 5 & 1.075 & $0.824-1.401$ & 0.595 & Random & 9.92 & 0.042 & $59.7 \%$ \\
\hline Recessive (TT versus CT + CC) & 5 & 1.104 & $0.805-1.516$ & 0.539 & Fixed & 2.92 & 0.572 & $0.0 \%$ \\
\hline \multicolumn{9}{|c|}{ XRCC1 gene Arg194Trp polymorphism in Caucasians populations } \\
\hline Allelic ( $\mathrm{T}$ versus $\mathrm{C}$ ) & 2 & 1.707 & $0.646-4.512$ & 0.281 & Random & 12.84 & 0.000 & $92.2 \%$ \\
\hline Heterozygous (CT versus CC) & 2 & 1.840 & $0.587-5.768$ & 0.295 & Random & 14.56 & 0.000 & $93.1 \%$ \\
\hline Homozygous (TT versus CC) & 2 & 1.815 & $0.521-6.321$ & 0.349 & Fixed & 0.20 & 0.656 & $0.0 \%$ \\
\hline Dominant $(\mathrm{TT}+\mathrm{CT}$ versus $\mathrm{CC})$ & 2 & 1.825 & $0.600-5.546$ & 0.289 & Random & 14.46 & 0.000 & $93.1 \%$ \\
\hline
\end{tabular}


Table 2 (continued)

\begin{tabular}{|c|c|c|c|c|c|c|c|c|}
\hline \multirow[t]{2}{*}{ Comparisons } & \multirow[t]{2}{*}{ No. of Studies } & \multicolumn{3}{|c|}{ Test of association } & \multirow[t]{2}{*}{ Analysis model } & \multicolumn{3}{|c|}{ Test of heterogeneity } \\
\hline & & OR & $95 \% \mathrm{CI}$ & P-value & & $x^{2}$ & P-value & $\mathrm{I}^{2}(\%)$ \\
\hline Recessive (TT versus CT + CC) & 2 & 1.607 & $0.463-5.584$ & 0.455 & Random & 0.08 & 0.775 & $0.0 \%$ \\
\hline \multicolumn{9}{|c|}{ XRCC1 gene Arg280His polymorphism in total populations } \\
\hline Allelic (A versus G) & 3 & 0.956 & $0.589-1.550$ & 0.854 & Random & 8.40 & 0.015 & $76.2 \%$ \\
\hline Heterozygous (GA versus GG) & 3 & 0.772 & $0.592-1.008$ & 0.057 & Fixed & 2.97 & 0.227 & $32.6 \%$ \\
\hline Homozygous (AA versus GG) & 3 & 1.216 & $0.630-2.349$ & 0.560 & Fixed & 3.60 & 0.165 & $44.5 \%$ \\
\hline Dominant (AA + GA versus GG) & 3 & 0.885 & $0.565-1.385$ & 0.592 & Random & 5.86 & 0.053 & $65.9 \%$ \\
\hline Recessive (AA versus GA + GG) & 3 & 1.261 & $0.653-2.432$ & 0.490 & Fixed & 3.07 & 0.215 & $34.9 \%$ \\
\hline \multicolumn{9}{|c|}{ XRCC1 gene Arg280His polymorphism in HWE } \\
\hline Allelic (A versus G) & 2 & 0.743 & $0.576-0.958$ & 0.022 & Fixed & 0.04 & 0.833 & $0.0 \%$ \\
\hline Heterozygous (GA versus GG) & 2 & 0.701 & $0.525-0.936$ & 0.016 & Fixed & 0.00 & 0.996 & $0.0 \%$ \\
\hline Homozygous (AA versus GG) & 2 & 0.794 & $0.339-1.859$ & 0.595 & Fixed & 0.98 & 0.322 & $0.0 \%$ \\
\hline Dominant (AA + GA versus GG) & 2 & 0.710 & $0.537-0.939$ & 0.016 & Fixed & 0.03 & 0.873 & $0.0 \%$ \\
\hline Recessive (AA versus GA + GG) & 2 & 0.856 & $0.367-1.999$ & 0.720 & Fixed & 0.90 & 0.342 & $0.0 \%$ \\
\hline \multicolumn{9}{|c|}{$\mathrm{XRCC} 1$ gene $\mathrm{c} .1517 \mathrm{G}>\mathrm{C}$ polymorphism in total populations } \\
\hline Allelic (C versus $\mathrm{G})$ & 2 & 1.252 & $1.064-1.473$ & 0.007 & Fixed & 0.52 & 0.471 & $0.0 \%$ \\
\hline Heterozygous (GC versus GG) & 2 & 1.025 & $0.786-1.335$ & 0.858 & Fixed & 0.01 & 0.903 & $0.0 \%$ \\
\hline Homozygous (CC versus GG) & 2 & 1.800 & $0.976-3.322$ & 0.060 & Random & 2.41 & 0.120 & $58.6 \%$ \\
\hline Dominant (CC + GC versus GG) & 2 & 1.187 & $0.924-1.526$ & 0.180 & Fixed & 0.00 & 0.973 & $0.0 \%$ \\
\hline Recessive (CC versus GC + GG) & 2 & 1.677 & $0.876-3.211$ & 0.119 & Random & 4.63 & 0.031 & $78.4 \%$ \\
\hline \multicolumn{9}{|c|}{ XRCC1 gene c. $1471 \mathrm{G}>$ A polymorphism in total populations } \\
\hline Allelic (A versus G) & 2 & 0.914 & $0.467-1.789$ & 0.794 & Random & 15.61 & 0.000 & $93.6 \%$ \\
\hline Heterozygous (GA versus GG) & 2 & 0.948 & $0.547-1.641$ & 0.848 & Random & 5.51 & 0.019 & $81.9 \%$ \\
\hline Homozygous (AA versus GG) & 2 & 0.860 & $0.210-3.513$ & 0.833 & Random & 12.31 & 0.000 & $91.9 \%$ \\
\hline Dominant (AA + GA versus GG) & 2 & 0.915 & $0.444-1.886$ & 0.810 & Random & 10.66 & 0.001 & $90.6 \%$ \\
\hline Recessive (AA versus GA + GG) & 2 & 0.879 & $0.277-2.789$ & 0.827 & Random & 8.92 & 0.003 & $88.8 \%$ \\
\hline
\end{tabular}

\section{Discussion}

Emerging evidences have shown that common genetic polymorphisms in XRCC1 gene may be associated with the development of pancreatic cancer, but individually published studies and previous meta-analyses revealed inconclusive results. In the present study, to obtain a more precise estimation of the association between the XRCC1 gene polymorphisms and pancreatic cancer risk, we conducted a systematic review with meta-analysis by critically reviewing all published studies.

For XRCC1 gene Arg399Gln polymorphism, we found no strong evidence of association with susceptibility to pancreatic cancer. Wang et al. [17] study was considered as deviation from HWE. We performed a stratified analysis based on HWE, and the pooled data also demonstrated that there was no evidence of association between the Arg399Gln polymorphism and the susceptibility of pancreatic cancer. We performed a subgroup analysis based on ethnicity, and the pooled data also demonstrated that there was no evidence of association between the Arg399Gln polymorphism and the susceptibility of pancreatic cancer in Asians or Caucasians. However, it was not in agreement with the result of Jiang et al. [18] study in which the pooled data demonstrated that XRCC1 gene Arg399Gln (rs25487) polymorphism is associated with pancreatic cancer risk in Asians. Sensitivity analysis demonstrated that pooled ORs were not materially altered, indicating that our results were robust and reliable. After evaluating the publication bias by Begg's funnel plots and
Table 3 Results of publication bias by Egger's linear regression test for the Arg399GIn polymorphism

\begin{tabular}{llllll}
\hline Egger's test & A versus G & GA versus GG & AA versus GG & AA + GA versus GG & AA versus GA + GG \\
\hline $\mathrm{t}$ & 0.34 & 0.06 & 0.25 & 0.19 & 0.23 \\
$P$-value & 0.746 & 0.955 & 0.812 & 0.854 & 0.824 \\
\hline
\end{tabular}


Egger's regression method, we did not detect a publication bias based on total population, indicating the strength of the results.

In this meta-analysis, a total of 7 case-control studies were analyzed to provide a comprehensive assessment of the association between Arg194Trp polymorphism and pancreatic cancer risk. Our results did not support a genetic association between Arg194Trp and susceptibility to pancreatic cancer. Neither allele frequency nor genotype distribution was significantly associated with susceptibility to pancreatic cancer. Since the incidence of gene polymorphisms may vary between different ethnic groups and this variation may interfere with the detection of minor effect of SNPs on pancreatic cancer risk, subgroup analysis according to ethnicity was performed to further explore the potential association between Arg194Trp and the risk of pancreatic cancer. However, even within the same ethnic group, no association of statistical significance was observed.

For XRCC1 gene Arg280His polymorphism, 3 casecontrol studies including 918 cases and 1147 controls were included in our systematic review. Wang et al. [17] study was considered as deviation from HWE. Results from our stratified analysis based on HWE showed that there was a robust significant association between Arg280His polymorphism and pancreatic cancer risk. However, previous meta-analysis conducted by Shen et al. [19] reported there was no evidence that XRCC1 gene Arg280His polymorphism are associated with pancreatic cancer risk. Their meta-analysis with only one eligible study [20] has insufficient statistical power to detect a small effect, while our meta-analysis included 3 casecontrol studies including 918 cases and 1147 controls. So the reasons for inconsistent results might be that larger sample sizes may lead to the identification of statistically significant correlation.

Two case-control studies on the relationship between c. $1517 \mathrm{G}>\mathrm{C}$ polymorphism and susceptibility to pancreatic cancer were included in our systematic review. Zhao et al. [21] and Hou et al. [22] reported that the XRCC1 c. $1517 \mathrm{G}>\mathrm{C}$ variant is significantly associated with pancreatic cancer susceptibility in the Chinese population. According to the current evidence-based literature, we thought that there was significant association between XRCC1 gene c. $1517 \mathrm{G}>\mathrm{C}$ polymorphism and pancreatic cancer risk.

Similarly, Two case-control studies on the relationship between c. $1471 \mathrm{G}>$ A polymorphism and susceptibility to pancreatic cancer were included in our meta-analysis. Retracted article conducted by Chen et al. [23] reported that the AA genotype was associated with the decreased risk of developing pancreatic cancer compared to GG wild genotype. Hou et al. [22] reported that the variant A allele frequency was borderline higher among patients in the pancreatic cancer group compared with the control group. To our surprise, we failed to find any association between c. $1471 \mathrm{G}>\mathrm{A}$ polymorphism and the risk for pancreatic cancer in any of the examined genetic models. For the limited studies, the results should be treated with caution.

However, several limitations of this meta-analysis should be considered. Firstly, our exclusion of papers published in languages other than English and Chinese might have biased our results. Secondly, the sample size is not large enough, especially for subgroup analysis. Thus, the observed significant associations in some subgroup analysis may be not accurate. Lastly, lack of available data prevented us from performing additional subgroup analyses by age, gender, alcohol consumption and other risk factors, which could be potential factors influencing the evaluation of the associations between SNPs in XRCC1 gene and pancreatic cancer risk.

In conclusion, the present meta-analysis suggested that Arg280His and c. $1517 \mathrm{G}>\mathrm{C}$ polymorphisms in XRCC1 gene were associated with pancreatic cancer risk. Considering the limited sample size and ethnicity enrolled in this meta-analysis, further larger scaled studies should be performed to validate the association.

Funding The authors have no support or funding to report.

\section{Compliance with Ethical Standards}

Conflict of Interest The authors report no conflicts of interest in this work.

Open Access This article is distributed under the terms of the Creative Commons Attribution 4.0 International License (http:// creativecommons.org/licenses/by/4.0/), which permits unrestricted use, distribution, and reproduction in any medium, provided you give appropriate credit to the original author(s) and the source, provide a link to the Creative Commons license, and indicate if changes were made.

\section{References}

1. Vaccaro V, Gelibter A, Bria E, Iapicca P, Cappello P, Di Modugno F et al (2012) Molecular and genetic bases of pancreatic cancer. Curr Drug Targets 13(6):731-743

2. Krejs GJ (2010) Pancreatic cancer: epidemiology and risk factors. Dig Dis 28(2):355-358. https://doi.org/10.1159/000319414

3. Ginsberg G, Angle K, Guyton K, Sonawane B (2011) Polymorphism in the DNA repair enzyme XRCC1: utility of current database and implications for human health risk assessment. Mutat Res Fundam Mol Mech Mutagen 727(1-2):1-15

4. Wood RD, Mitchell M, Sgouros J, Lindahl T (2001) Human DNA repair genes. Science 291(5507):1284-1289

5. Tudek B (2007) Base excision repair modulation as a risk factor for human cancers. Mol Asp Med 28(3-4):258-275. Epub 2007/07/14. https://doi.org/10.1016/j.mam.2007.05.003

6. Vidal AE, Boiteux S, Hickson ID, Radicella JP (2001) XRCC1 coordinates the initial and late stages of DNA abasic site repair through protein-protein interactions. EMBO J 20(22):6530-6539

7. Feng YZ, Liu YL, He XF, Wei W, Shen XL, Xie DL (2014) Association between the XRCC1 Arg194Trp polymorphism and risk of cancer: evidence from 201 case-control studies. Tumour 
biology: the journal of the International Society for Oncodevelopmental Biology and Medicine 35(11):10677-10697. https://doi.org/10.1007/s13277-014-2326-x

8. Li J, Li Z, Feng L, Guo W, Zhang S (2013) Polymorphisms of DNA repair gene XRCC1 and hepatocellular carcinoma risk among east Asians: a meta-analysis. Tumour biology: the journal of the International Society for Oncodevelopmental Biology and Medicine 34(1):261-269. https://doi.org/10.1007/s13277-012-0546-5

9. Dai L, Duan F, Wang P, Song C, Wang K, Zhang J (2012) XRCC1 gene polymorphisms and lung cancer susceptibility: a metaanalysis of 44 case-control studies. Mol Biol Rep 39(10):95359547. https://doi.org/10.1007/s11033-012-1818-2

10. Moher D, Liberati A, Tetzlaff J, Altman DG (2009) Preferred reporting items for systematic reviews and meta-analyses: the PRISMA statement. PLoS Med 6(7):e1000097. https://doi.org/10. 1371/journal.pmed.1000097

11. Rohlfs RV, Weir BS (2008) Distributions of hardy-Weinberg equilibrium test statistics. Genetics 180(3):1609-1616. https://doi.org/ 10.1534/genetics.108.088005

12. Huedo-Medina TB, Sanchez-Meca J, Marin-Martinez F, Botella J (2006) Assessing heterogeneity in meta-analysis: Q statistic or I2 index? Psychol Methods 11(2):193-206. https://doi.org/10.1037/ 1082-989x.11.2.193

13. DerSimonian R, Laird N (1986) Meta-analysis in clinical trials. Control Clin Trials 7(3):177-188. https://doi.org/10.1016/01972456(86)90046-2

14. Mantel N, Haenszel W (1959) Statistical aspects of the analysis of data from retrospective studies of disease. J Natl Cancer Inst 22(4): 719-748

15. Begg CB, Mazumdar M (1994) Operating characteristics of a rank correlation test for publication bias. Biometrics 50(4):1088-1101
16. Egger M, Davey Smith G, Schneider M, Minder C (1997) Bias in meta-analysis detected by a simple, graphical test. BMJ (Clinical research ed) 315(7109):629-634

17. Wang LJ, Wang HT, Wang XX (2016) Association of XRCC1 gene polymorphisms and pancreatic cancer risk in a Chinese population. Genet Mol Res 15(2):gmr8080

18. Jiang H, Wu D, Ma D, Lin G, Liang J, Jin J (2013) Association between X-ray repair cross-complementation group 1 rs 25487 polymorphism and pancreatic cancer risk. Tumor Biol 34(6): 3417-3421

19. Shen WD, Chen HL, Liu PF (2011) XRCC1 polymorphisms and pancreatic cancer: a meta-analysis. Chin J Cancer Res 23(3):165170. https://doi.org/10.1007/s11670-011-0165-5

20. Mcwilliams RR, Bamlet WR, Cunningham JM, Goode EL, Andrade MD, Boardman LA et al (2008) Polymorphisms in DNA repair genes, smoking, and pancreatic adenocarcinoma risk. Cancer Res 68(12):4928-4935

21. Zhao ZM, Li CG, Hu MG, Gao YX, Liu R (2014) Influence of the $c .1517 \mathrm{G}>\mathrm{C}$ genetic variant in the XRCC1 gene on pancreatic cancer susceptibility in a Chinese population. Genet Mol Res 13(2):4466-4472. https://doi.org/10.4238/ 2014.June.16.5

22. Hou BH, Jian ZX, Cui P, Li SJ, Tian RQ, Ou JR (2016) Association and intragenic single-nucleotide polymorphism interactions of the XRCC1 polymorphisms for pancreatic cancer susceptibility. Pancreas 45(4):546-551. https://doi.org/10.1097/mpa. 0000000000000482

23. Chen H, Tang C, Liu M, Zhou B, Kuang Y, Yuan T et al (2013) Association of XRCC1 gene single nucleotide polymorphisms and susceptibility to pancreatic cancer in Chinese. Tumor Biol 35(1): 27-32. https://doi.org/10.1007/s13277-013-1001-y 